2006-01-01

\title{
Evidence of multimodal structure of the baroclinic tide in the Strait of Gibraltar
}

\author{
Vazquez, A
}

http://hdl.handle.net/10026.1/3857

10.1029/2006gl026806

Geophysical Research Letters

American Geophysical Union (AGU)

All content in PEARL is protected by copyright law. Author manuscripts are made available in accordance with publisher policies. Please cite only the published version using the details provided on the item record or document. In the absence of an open licence (e.g. Creative Commons), permissions for further reuse of content should be sought from the publisher or author. 


\title{
Evidence of multimodal structure of the baroclinic tide in the Strait of Gibraltar
}

\author{
A. Vázquez, ${ }^{1}$ N. Stashchuk, ${ }^{2}$ V. Vlasenko, ${ }^{2}$ M. Bruno, ${ }^{1}$ A. Izquierdo, ${ }^{1}$ and P. C. Gallacher ${ }^{3}$ \\ Received 4 May 2006; revised 6 July 2006; accepted 20 July 2006; published 8 September 2006.
}

[1] The multimodal structure of the baroclinic tides in the Strait of Gibraltar is studied using observations and numerical simulations. Observational data and model results revealed the presence of two types of tidal internal waves generated over Camarinal Sill (CS). One propagates toward the Mediterranean and disintegrates into series of nonlinear short internal waves with amplitudes of $50 \mathrm{~m}$ and more. The second type, behind the first, propagates slower and has a longer wavelength. The vertical structure with both upward and downward displacements of isopycnals can be identified as a manifestation of higher baroclinic modes. Analysis of the empirical orthogonal functions of the ADCP measurements performed over CS and model time series has shown that the second baroclinic mode predominates in the second type of internal waves. Its amplitude can reach one third that of the first baroclinic mode of the leading waves of depression. Citation: Vázquez, A., N. Stashchuk, V. Vlasenko, M. Bruno, A. Izquierdo, and P. C. Gallacher (2006), Evidence of multimodal structure of the baroclinic tide in the Strait of Gibraltar, Geophys. Res. Lett., 33, L17605, doi:10.1029/2006GL026806.

\section{Introduction}

[2] The Strait of Gibraltar has a great impact on the environment of the Mediterranean Sea and Atlantic Ocean. It can make an important contribution to the formation of the global oceanic circulation by supplying nutrient-rich heavy salty waters from the Mediterranean Sea to the Atlantic Ocean via the Mediterranean Outflow (MO) [Rahmstorf, 1997; Reid, 1979].

[3] The circulation in the Strait of Gibraltar is roughly characterized by a two-layer structure with Atlantic waters (less salty and warmer) flowing into the Mediterranean in the upper layer and the Mediterranean waters (saltier and colder) flowing out below [Armi and Farmer, 1988; Tsimplis, 2000]. The density of the final MO penetrating into the Atlantic Ocean and forming the Mediterranean waters, is determined by the mixing and entrainment processes at small scale in the strait. The density of the Atlantic water inflowing into the Alboran Sea from the strait, must also be affected by these processes.

[4] One of the most important mechanisms for mixing in the Global Ocean is internal waves. Particularly, in the Strait

\footnotetext{
${ }^{1}$ Departamento de Física Aplicada, Facultad de Ciencias del Mar y Ambientales, Universidad de Cádiz, Puerto Real, Spain.

${ }^{2}$ School of Earth, Ocean and Environmental Science, University of Plymouth, Plymouth, UK.

${ }^{3}$ Naval Research Laboratory, Stennis Space Center, Mississippi, USA.
}

Copyright 2006 by the American Geophysical Union. 0094-8276/06/2006GL026806\$05.00 of Gibraltar high amplitude internal waves associated with the internal tidal dynamics have been recognized as the main contributors to the mixing between Atlantic and Mediterranean layers [Wesson and Gregg, 1988; Bruno et al., 2002; Macias et al., 2006]. This strait is known as one of the most famous "hot spots" of the World Ocean where large amplitude internal waves are regularly observed [see, e.g., Armi and Farmer, 1988; Farmer and Armi, 1988; Wesson and Gregg, 1994; Pettigrew and Hyde, 1990; Watson and Robinson, 1990; Bray et al., 1990; Watson, 1994; Richez, 1994; Pistek and La Violette, 1999; Tsimplis, 2000; Izquierdo et al., 2001; Bruno et al., 2002]. These waves are generated by tidal flow interacting with Camarinal Sill (CS) which is located near the western entrance to the Strait of Gibraltar (see Figure 1e). According to the present understanding, an interfacial depression is formed over the western flank of CS when the tidal current is flowing toward the Atlantic Ocean (outflow). This depression, being arrested over CS by strong tidal flow, is released and begins to move eastward when the outflowing current slackens. During its propagation towards the Mediterranean Sea, the baroclinic bore disintegrates into a series of nonlinear short internal waves. As the interfacial disturbances arrive to Tarifa Narrows (TN) (see Figure 1e), the tidal current starts to flow eastward. As a result another weaker baroclinic bore is formed over the eastern flank of CS. It propagates westward as soon as tidal flux weakens.

[5] Most of the existing works devoted to the study of the baroclinic tidal response of the Strait of Gibraltar have focused on the analysis of these waves, which in fact demonstrate an overwhelming first-mode baroclinic signal. However, the measurements by Armi and Farmer [1988] and Farmer and Armi [1988], as well as observation by Wesson and Gregg [1988] also revealed evidence of a higher baroclinic mode signal, which has not been described yet by any theoretical model. In this paper we present a new experimental data set which shows evidence of the multimodal structure of baroclinic tides in the Strait of Gibraltar. These data are compared with the results of a fully nonlinear, nonhydrostatic numerical model. Finally, an Empirical Orthogonal Function (EOF) analysis is applied to the model and measured velocity data in order to quantify the modal structure of the baroclinic waves.

\section{Experimental Data}

[6] The experimental data used in this paper were collected on different cruises during the GIMIX project. Two typical along-strait temperature sections showing the multimodal structure of baroclinic tides over CS are presented in Figure 1. They were built on the basis of an XBT survey performed from the vessel moving with constant velocity 

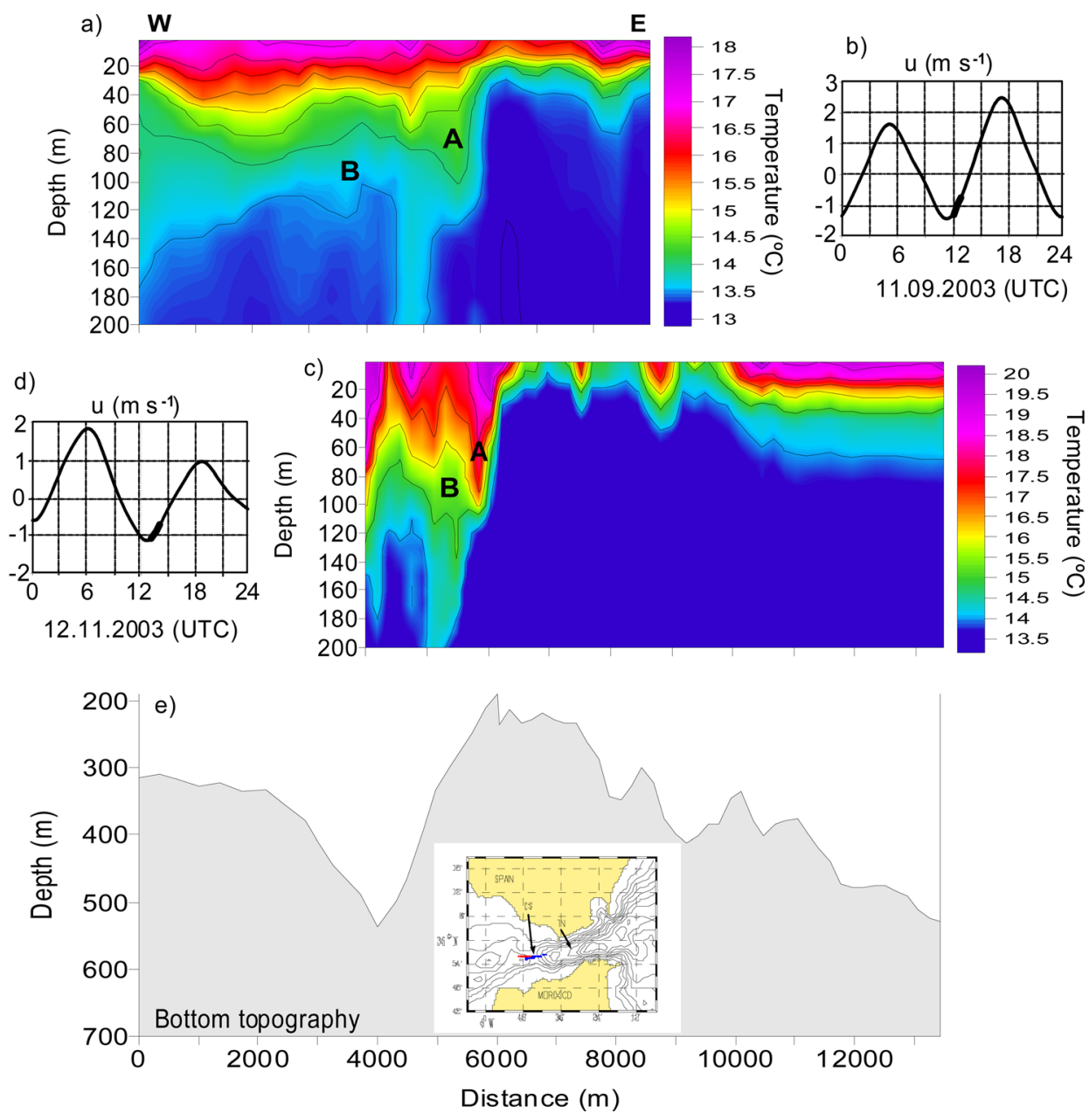

Figure 1. Temperature transects across CS obtained on (a) 11.09.03 and (c) 12.11.03, (b and d) the associated tidal current predictions over CS, and (e) bottom topography of CS and the map of the Strait of Gibraltar with indication of the transects for Figure 1a (red line) and $1 \mathrm{~b}$ (blue line). TN denotes the Tariffa Narrows.

over Camarinal Sill. Figure 1a was constructed from 34 temperature profiles taken between 12:18 and 14:04 UTC on the 11th September 2003 with a mean spatial resolution of $270 \mathrm{~m}$. The second transect (Figure 1c) was performed between 13:01 and 13:51 UTC on the 12th November 2003. In the last case 52 XBT profiles were taken with a mean spatial resolution of $170 \mathrm{~m}$. Figures $1 \mathrm{~b}$ and $1 \mathrm{~d}$ show the prediction of the along strait component of tidal velocity over CS during the XBT measurements. This prediction was performed on the basis of a harmonic analysis using 38 harmonic components of velocity at $45 \mathrm{~m}$ depth registered over the CS from October of 1994 to April of 1995. In a near-bottom layer the temporal variability of the currents is also characterized by tidal periodicity [Garcia Lafuente et al., 2000]; however, the absolute value of velocity here is different due to the two layer structure of the circulation in the strait described above.

[7] Although the temperature transects were obtained from different cruises, they reveal quite similar structure. Both of them were taken approximately 1 hour after the maximum outflow of the current when internal waves have been generated over CS (find thick segments in Figures 1b and 1d). The areas covered by Figures $1 \mathrm{a}$ and $1 \mathrm{c}$ are not identical although both include the area just above the sill.
The comparative analysis of two temperature fields, measured just after the maximum outflow, allows one to conclude that qualitatively they are quite similar even though they were taken two month apart. At the east side of CS the vertical temperature structure is almost undisturbed (Figure 1c) whereas just above the sill's crest the isotherms are elevated and some of them even outcrop. A complex structure of the temperature field was located to the west of the CS. Superposition of several baroclinic modes at the lee side of the CS is evident (find, for instance, the fragment $\mathrm{A}$, with all isotherms displaced downward, and the fragment B, with isotherms displaced upward and downward, these can be identified as first and second-mode waves, respectively). This pattern in general coincides with the observations obtained in November 2003 (Figure 1c) where similar waves, $\mathrm{A}$ and $\mathrm{B}$, can be identified. It can be seen that the vertical scale of the isotherms displacements exceeds $100 \mathrm{~m}$ behind the sill. The $14^{\circ} \mathrm{C}$ isotherm is displaced as much as $200 \mathrm{~m}$ which, to the best of our knowledge, may be the largest internal wave amplitude ever recorded.

[8] Although we are using temperature as a tracer to delineate the structure of the internal waves, we are not implying that the temperature gradient necessarily generates 
the restoring force for the waves. Salinity can be very important in determining the density structure, hence the buoyancy, in the strait. However the isotherms accurately reflect the displacement of the water, hence the amplitude of the internal waves.

[9] The temperature gradients here are weaker in comparison with the layer of seasonal thermocline. As it will be shown below from the analysis of the boundary value problem, the maximum wave displacement is expected at the depth of about $100 \mathrm{~m}$ which roughly coincides with undisturbed position of the $14^{\circ} \mathrm{C}$ isotherm.

[10] Another important conclusion from the observational data is that the multi-modal nature of baroclinic tide in the area of CS is clearly evident. At first glance these higher mode waves (packet B) may be thought to be a mixing area produced by the first baroclinic mode waves [Wesson and Gregg, 1988]. However, this structure is not stationary, as will be shown below. It has a phase speed, which is different from that of the first mode wave. It propagates eastward along the Strait of Gibraltar revealing that it is a progressive wave. The study of the complex multi-modal nature of the phenomenon is the topic of the rest of this paper.

\section{Numerical Modeling}

[11] A number of papers have been published during last two decades on numerical modeling of the baroclinic dynamics of the Strait of Gibraltar. All attempts to model the baroclinic tidal dynamics in the strait, from the first application of a three-dimensional ocean circulation model by Wang [1989] until now, failed to describe the real characteristics of generated waves because of the "hydrostatic" approximation used in most of them (see also the very recent paper by Sannino et al. [2004]). These difficulties were partly overcome in works by Brandt et al. [1996] and Morozov et al. [2002], where nonhydrostatic effects were incorporated in the models allowing for description of the structure and dynamics of a baroclinic tidal bore. However, the detailed structure of higher mode baroclinic modes was not studied in these papers, because the model by Brandt et al. [1996] was two-layered and numerical runs by Morozov et al. [2002] were performed on a coarse horizontal grid (200 m grid step).

[12] In this paper a fully nonlinear non-hydrostatic $\sigma$ coordinate numerical model was used to reproduce the baroclinic tide generated over the CS. The model is $2.5 \mathrm{D}$ because the equations are laterally averaged to account for variations in the cross-section of the strait. Details of the model are described by Vlasenko et al. [2005]. The calculation domain extended longitudinally from $5.09^{\circ} \mathrm{W}$ to $6.10^{\circ} \mathrm{W}$, covering a total of $90 \mathrm{~km}$. The horizontal grid step was $50 \mathrm{~m}$ and $140 \sigma$-levels were used in the vertical dimension. The bottom topography of the Strait of Gibraltar, shown in Figure 1e, was used in the model. The topography was slightly smoothed to prevent small scale noise from developing in the simulations (compare the bottom plots in Figures 1 and 2). Initial temperature and salinity profiles were taken from real CTD measurements. The fluid was initially at rest.

[13] The model was forced by the M2 tidal current during three semi-diurnal tidal cycles. Figure 2 shows the model predicted evolution of the density field in the area of CS during one tidal cycle. The run starts at $t=0 \mathrm{~h}$, when the current is zero at CS changing from inflow (eastward) to outflow (westward). During the first two hours of the tidal cycle the accelerating barotropic flow produces a weak elevation of isopycnals upstream of the crest of CS and a deep isopycnal depression $(\sim 50 \mathrm{~m})$ at the lee side of it. This wave of depression is arrested by the accelerating barotropic flux behind the obstacle where it extracts energy from the background flow and grows in amplitude due to the permanent feedback with the barotropic flow.

[14] In fact, the initial wave at this stage can be treated as a lee wave propagating upstream (the inclined line c-c represents the line of equal phase). Normally the arrested lee waves possess characteristics of both first and higher baroclinic modes (all isopycnal displacements are downward in section a-a and isopycnal displacements are both upward and downward in section b-b). The first and higher modes possess different phase velocities thus they effectively separate during propagation. This process of separation is clearly seen in Figure 2. By $t=3 \mathrm{~h}$ the fastest first-mode baroclinic bore separates from the rest of the wave field. As a result of the separation, two structures of isopycnal displacements can be distinguished at time $t=3-$ $4 \mathrm{~h}$, resembling the structures observed in the XBT transects (Figure 2, shaded areas). The first-mode baroclinic bore (A), in which the isopycnals are depressed toward the bottom in the whole water column, is located just over the Camarinal Sill. The second structure (wave B) represents the 2-nd baroclinic mode with isopycnals displaced upward above the pycnocline and downward below it. The scope of vertical isopycnal displacements (from trough to crest) is comparable to that found in the temperature measurements. When the outflow current slackens $(t=4 \mathrm{~h})$, bore A starts to move eastward disintegrating into a packet of first-mode nonlinear internal waves while the rest of the wave field, B, is still retained at the west side of the CS. It is released at $t=$ $6 \mathrm{~h}$ when the current reverses and starts to generate a new system of waves propagating westward (packet C).

[15] Figure 2 shows the first tidal cycle. Thus, initial transient processes may influence the results shown in Figure 2 . We deliberately chose the beginning of the motion to show the generation mechanism more clearly (from the initial cycle, when the wave field is not contaminated by other processes). Note, however, that for subsequent tidal cycles the mechanism of the waves generation and evolution qualitatively remains the same, although the structure of the waves field can be slightly different in details.

[16] The mean phase speed of the baroclinic bore (A) in the numerical experiment was about $1.40 \mathrm{~m} \mathrm{~s}^{-1}$, which is in agreement with the values computed by Izquierdo et al. [2001] and Sannino et al. [2004]. At the same time the phase speed of the structure B in the numerical model was about $0.60 \mathrm{~m} \mathrm{~s}^{-1}$, which is less than half of the phase speed of the wave A. Similar values of the propagation speed of second baroclinic mode for this area were observed by Armi and Farmer [1988].

\section{EOF Analysis}

[17] An empirical orthogonal function (EOF) analysis of the time series of vertical velocity obtained from observations and numerical simulations was carried out to get more 

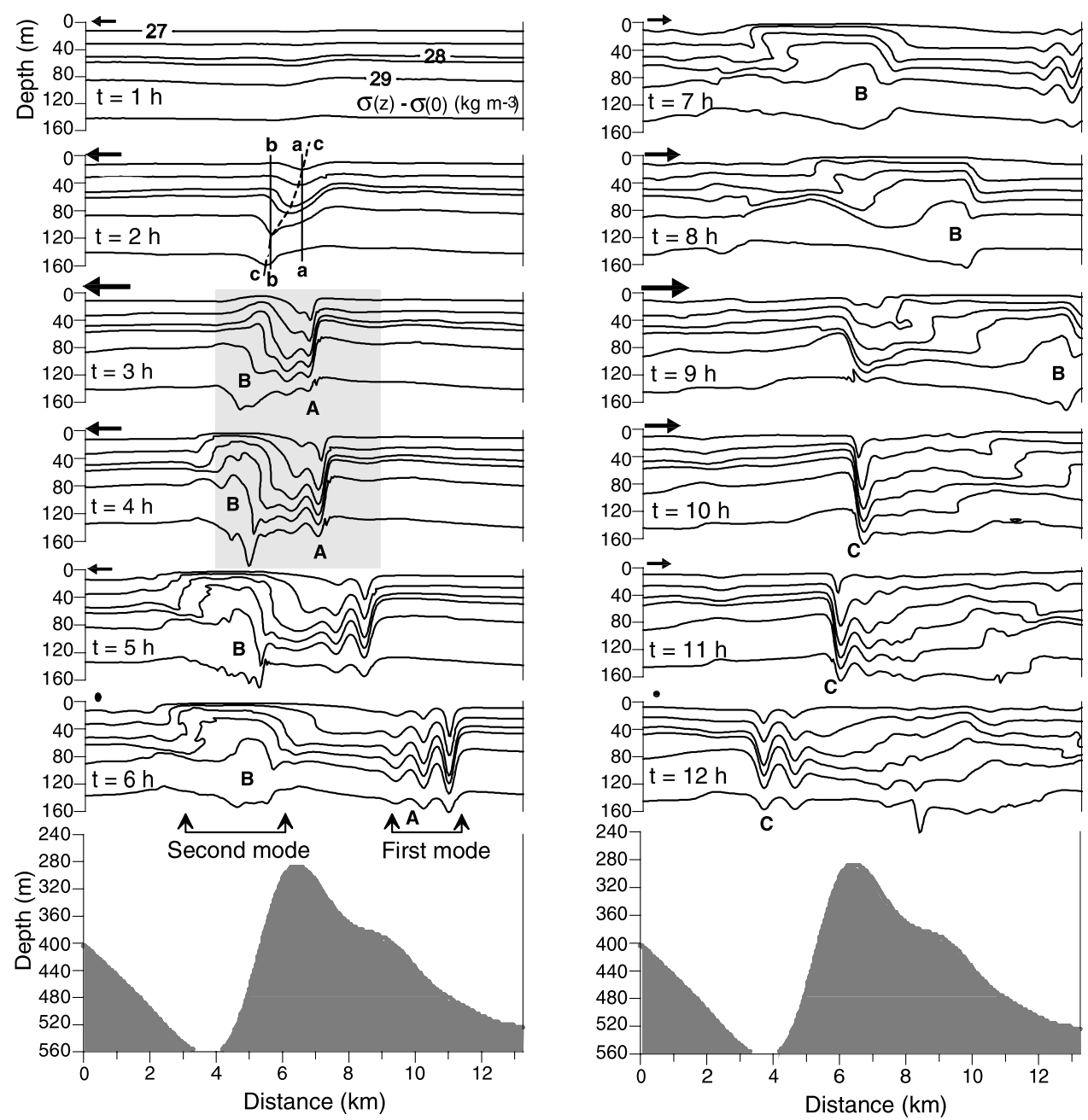

Figure 2. Model predicted evolution of the density field during one tidal cycle. The arrows show the relative intensity and direction of the tidal current. Letters A, B and C denote particular fragments of the wave field. The shaded areas at the bottom represent the smoothed topography of CS.

detailed information about the structure of the generated waves. The vertical structure of currents over CS was measured by a $190 \mathrm{KHz}$ Acoustic Doppler Profiler (ADP) during 25 hours on 10th November 2003. The temporal resolution of these measurements was $60 \mathrm{~s}$, and the bin size was equal to $2.5 \mathrm{~m}$.

[18] The structure of the first two empirical functions of model (Figure 3a) and ADP (Figure 3c) velocities are in
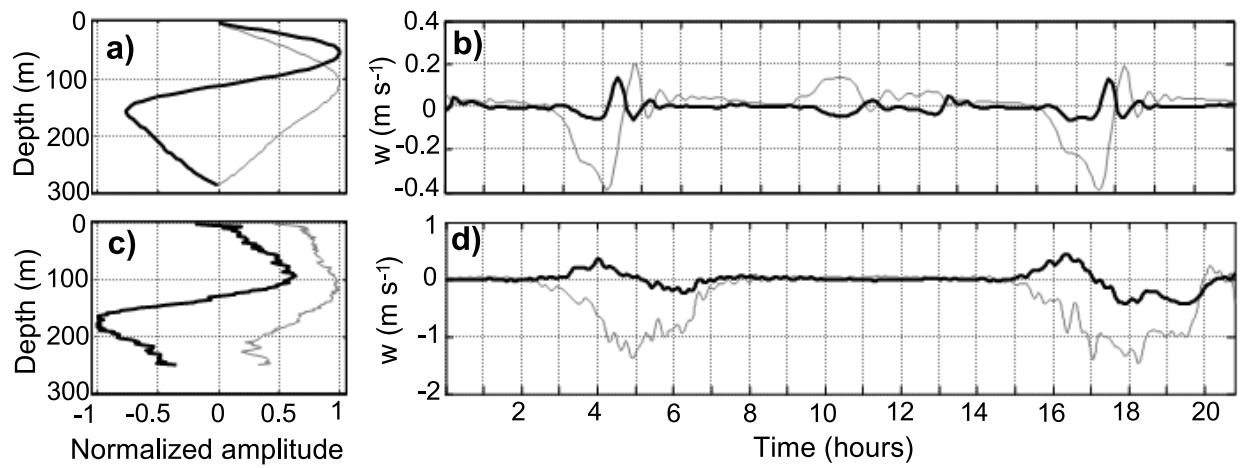

Figure 3. Characteristics of the first (gray lines) and second (black lines) modes over the top of Camarinal Sill. Normalized amplitudes of EOFs calculated from model (a) and ADP (c) vertical velocities. Appropriate time series of vertical velocity amplitude (at the depths where the EOFs have maximum) calculated from (b) the model results and (d) the experimental data. 
qualitative agreement and resemble the first and second dynamical, modes, $g_{j}(z)$ obtained from the boundary value problem

$$
\begin{gathered}
g_{z z}+(N(z) / c)^{2} g=0, \\
g(0)=g(-H)=0 .
\end{gathered}
$$

Here $N(z)$ is the buoyancy frequency, $c$ is the phase speed, and $H$ is the water depth. The amplitude of the first mode does not change sign over the water column and, therefore, all the isopycnals oscillate in the same direction with maximum displacement at a depth of about $100 \mathrm{~m}$. The second EOF changes sign at a depth of about $130 \mathrm{~m}$ indicating that isopycnals are moving in opposite directions above and below the pycnocline. This behavior is in agreement with the second dynamical mode.

[19] The temporal variability of the maximum vertical velocity of the two first EOFs over CS are shown in Figure $3 \mathrm{~d}$ for the observational time series and in $3 \mathrm{~b}$ for the model time series. Time $t=0$ corresponds to the beginning of a tidal cycle when the background tidal flow is zero. The second tidal cycle (from $t=T$ ) was used for the EOF analysis of the numerical data to insure that the "spin-up" of the model was completed and no transient processes are present in Figure 3b. It should be noted that EOF analysis was carried out after the elimination of the temporal mean current from the vertical velocity series.

[20] Both numerical and experimental curves reveal evidence of quite strong first- and second-mode signals. An analysis of these time series shows that the maximum signal in both cases takes place 3-6 $\mathrm{h}$ after the beginning of the tidal cycle. As was demonstrated above by the numerical modeling, the generation of a strong depression of the pycnocline above CS, its separation into a first mode baroclinic bore (packet $\mathrm{A}$ ) and into the multi-modal wave structure (packet B) takes place during this time span.

[21] Some discrepancies between simulated and observed data can be explained in terms of the assumptions made in the model. For instance, they could be caused by the manifestation of 3D effects, which were not included in the model (except for the variable cross-section), or they could be caused by disregarding the mean flow through the strait. Another possibility could be the use of smoothed bathymetry in the model. However, apart from these discrepancies, one can conclude that EOF analysis of both theoretical and experimental series revealed qualitatively similar general patterns with evidence of the presence of strong secondmode signal. The vertical structure of the first two EOFs is very similar to those obtained from the boundary value problem. In conjunction with the data presented above from the XBT surveys and from the model results, the conclusions derived from the EOF analysis are strong evidence of the multimodal nature of baroclinic tide in the Strait of Gibraltar.

\section{Discussion and Summary}

[22] A strong semi-diurnal tidal forcing plays a fundamental role in the dynamics of the Strait of Gibraltar. This oscillatory flow interacts with Camarinal Sill creating internal waves which are the main source of energy for mixing and entrainment processes between the Atlantic inflow and the MO. The MO spreads into the eastern North-Atlantic forming a warm and salty tongue at intermediate depths, which is thought to contribute to the preconditioning of North Atlantic Deep Water formation, and hence to the global thermohaline circulation [Reid, 1979]. In addition, the mixing processes induced by the internal waves generated at the CS of the Strait of Gibraltar, modify the density of the Atlantic water jet entering the Alboran Sea [Macias et al., 2006].

[23] The analysis of observational data collected in the area of CS [Bruno et al., 2002] and the results of numerical modeling both revealed two types of large-amplitude internal waves with comparable amplitudes generated by tidal current. The first one, a packet of large amplitude internal solitary waves, is the result of disintegration of the well documented first-mode baroclinic bore generated by tidal flow over CS and propagating towards the Mediterranean. The second system of waves (behind the first) propagates slower and has relatively larger length scale. This system reveals the characteristics of quasi-steady higher baroclinic mode internal waves trapped by the flood flux just over the sill during the flood phase, lasting for $4 \mathrm{~h}$ (fragment $\mathrm{B}$ in Figure 2).

[24] From the observations this broadening of pycnocline might appear to be a mixing area produced by the first baroclinic mode waves in the areas of strong shear. However, the numerical modeling has shown that this structure is not stationary over the whole tidal cycle; it has a phase speed, and propagates eastward along the Strait of Gibraltar resembling a progressive wave behavior. The vertical structure of isopycnal displacement (Figure 3) along with the vertical structure of horizontal velocity obtained from the numerical runs (not shown here) clearly indicates that this disturbance can definitely be treated as progressive second mode wave. Thus, the experimental data presented here along with their theoretical interpretation can be considered as a strong evidence of the complex multimodal structure of baroclinic tide in the Strait of Gibraltar. Not only the wellknown first-mode internal waves but also higher mode baroclinic disturbances with comparable amplitude should be taken into account in a general balance of mixing processes in the strait.

[25] Acknowledgments. This study was carried out within NERC project NE/D007968/1. We would like to thank the Spanish research projects VEM2003-20577-CM-07 and REN2001-2733-C02-01. Patrick C. Gallacher was funded by the Naval Research Laboratory and ONR through project 73-6836-B6. We thank, as well, the Spanish Navy Hydrographic Institute for supplying the research vessels used in our initial field experiments. We are very thankful to two anonymous reviewers for their comments on the manuscript.

\section{References}

Armi, L., and D. Farmer (1988), The flow of the Mediterranean water through the Strait of Gibraltar, Prog. Oceanogr., 21, 1-105.

Brandt, P., W. Alpers, and J. O. Backhaus (1996), Study of the generation and propagation of internal waves in the Strait of Gibraltar using a numerical model and synthetic aperture radar images of the European ERS 1 satellite, J. Geophys. Res., 101, 14,237-14,252.

Bray, N. A., C. D. Winant, T. H. Kinder, and J. Candela (1990), Generation and kinematics of the internal tide in the Strait of Gibraltar, in The Physical Oceanography of Sea Strait, edited by L. J. Pratt, pp. 477491, Springer, New York.

Bruno, M., J. J. Alonso, A. Cózar, J. Vidal, A. Ruiz-Cañavate, F. Echevaria, and J. Ruiz (2002), The boiling-water phenomena at Camarinal Sill, the Strait of Gibraltar, Deep Sea Res., Part II, 49, 4097-4113.

Farmer, D., and L. Armi (1988), The flow of the Atlantic water through the Strait of Gibraltar, Prog. Oceanogr., 21, 1-105. 
Garcia Lafuente, J. G., J. M. Vargas, F. Plaza, T. Sarhan, J. Candela, and B. Bascheck (2000), Tide at the eastern section of the Strait of Gibraltar, J. Geophys. Res., 105, 14,197-14,213.

Izquierdo, A., L. Tejedor, D. V. Sein, J. O. Backhaus, P. Brandt, A. Rubino, and B. A. Kagan (2001), Control variability and internal bore evolution in the Strait of Gibraltar: A 2-D two-layer model study, Estuarine Coastal Shelf Sci., 53, 637-651.

Macias, D., C. M. Garcia, F. Echevarria, A. Vazquez, and M. Bruno (2006), Tidal induced variability of mixing processes on Camarinal Sill (Strait of Gibraltar): A pulsating event, J. Mar. Syst., 60, 177-192.

Morozov, E., K. Trulsen, M. Velarde, and V. Vlasenko (2002), Internal tides in the Strait of Gibraltar, J. Phys. Oceanogr., 32, 3193-3206.

Pettigrew, N. R., and R. A. Hyde (1990), The structure of the internal bore in the Strait of Gibraltar and its influence on the Atlantic inflow, in The Physical Oceanography of Sea Straits, edited by L. J. Pratt, pp. 493-508, Springer, New York.

Pistek, P., and P. E. La Violette (1999), Observations of the suppression of tide-generated nonlinear internal wave packets in the Strait of Gibraltar, J. Mar. Syst., 20, 113-128.

Rahmstorf, S. (1997), Risk of sea-change in the Atlantic, Nature, 388, $825-826$.

Reid, J. L. (1979), On the contribution of the Mediterranean Sea outflow to the Norwegian-Greenland Sea, Deep Sea Res., 26, 1199-1223.

Richez, C. (1994), Airborne synthetic aperture radar tracking of interna waves in the Strait of Gibraltar, Progr. Oceanogr., 33, 93-159.

Sannino, G., A. Bargagli, and V. Artale (2004), Numerical modeling of the semidiurnal tidal exchange through the Strait of Gibraltar, J. Geophys. Res., 109, C05011, doi:10.1029/2003JC002057.
Tsimplis, M. N. (2000), Vertical structure of tidal currents over Camarinal Sill at the Strait of Gibraltar, J. Geophys. Res., 105, 19,709-19,728.

Vlasenko, V., N. Stashchuk, and K. Hutter (2005), Baroclinic Tides, 351 pp., Cambridge Univ. Press, New York.

Wang, D.-P. (1989), Model of mean and tidal flow in the Strait of Gibraltar, Deep Sea Res., 36, 1535-1548.

Watson, G. (1994), Internal waves in a stratified shear flow: The Strait of Gibraltar, J. Phys. Oceanogr., 24, 509-517.

Watson, G., and I. S. Robinson (1990), A study of internal wave propagation in the Strait of Gibraltar using shore-based radar images, J. Phys. Oceanogr., 20, 374-395.

Wesson, J., and M. Gregg (1988), Turbulent dissipation in the Strait of Gibraltar and associated mixing: Small-scale turbulence and mixing in the ocean, in Proceedings of the 19th International Liege Colloquium on Ocean Hydrodynamics, edited by J. Nihoul and B. Jamart, pp. 201-212, Elsevier, New York.

Wesson, J. C., and M. C. Gregg (1994), Mixing at Camarinal Sill in the Strait of Gibraltar, J. Geophys. Res., 99, 9847-9878.

M. Bruno, A. Izquierdo, and A. Vázquez, Departamento de Física Aplicada, Facultad de Ciencias del Mar y Ambientales, Universidad de Cádiz, E-11510 Puerto Real, Spain. (miguel.bruno@uca.es; alfredo. izquierdo@uca.es; agueda.vazquez@uca.es)

P. C. Gallacher, Naval Research Laboratory, Code 7331 Stennis Space Center, MS 39529, USA. (gallacher@nrlssc.navy.mil)

N. Stashchuk and V. Vlasenko, School of Earth, Ocean and Environmental Science, University of Plymouth, Plymouth PL4 8AA, UK. (nstashchuk@plymouth.ac.uk; vvlasenko@plymouth.ac.uk) 